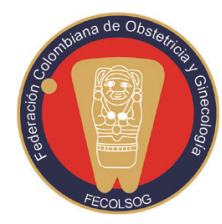

\title{
LA REVISTA COLOMBIANA DE OBSTETRICIA Y GINECOLOGÍA EN EL SIGLO XXI
}

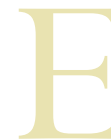
l siglo XXI ha traído cambios en muchos aspectos de la sociedad actual. Estos se presentan de diversas maneras, por ejemplo: en cómo nos comunicamos, en los estilos de vida, en las condiciones climáticas, en las preocupaciones de la población a corto y mediano plazo.

Hoy en día, la Internet es la principal vía de comunicación. Las redes sociales, como Twitter, son el camino más rápido para comunicar las noticias: este año, el alcalde electo de Bogotá (Colombia), publicó su gabinete por este medio (1). Los jóvenes utilizan Facebook como principal medio para comunicarse, y aplicaciones software (apps) como Skype, Hangouts o WhatsApp son cada vez más utilizadas por su facilidad y versatilidad, dado que pueden ser usadas desde las tabletas y los teléfonos inteligentes (2). En el campo de la salud, los pacientes tienen cada vez más interés por conocer aspectos del tratamiento y pronóstico del problema de salud que padecen, por lo que acceden a fuentes de información en la Web, así participan de manera más activa en las decisiones sobre el manejo de la misma. Por otra parte, en educación médica continuada, las conferencias vía Web cada vez son más utilizadas, en lugar o como complemento de las conferencias presenciales, ya que tienen el potencial de lograr mayores audiencias, se ajustan a diferentes horarios y permiten diferentes grados de interactividad (3).

En cuanto a los estilos de vida, cerca del $5 \%$ de los sujetos en Estados Unidos se identificaban en el 2012 como vegetarianos (4). Por otra parte, algunos estudios sugieren que las actividades en Internet y las aplicaciones para tabletas y teléfonos inteligentes compiten negativamente con la actividad física $(5,6)$. Estos patrones de baja actividad física, relacionados con alta dedicación de tiempo a la Internet y a juegos de consola, se acompañan de malos hábitos alimentarios y sobrepeso (7).

Es claro que el cambio climático está afectando al planeta (8), y cada vez se conocen más los efectos sobre las plantas (9), el mar (10) y la salud humana; por ejemplo, se han incrementado las áreas de infección del dengue y la malaria, y la disponibilidad de agua potable a nivel mundial se está viendo afectada (11).

De esta manera, surgen nuevos problemas por resolver: cómo frenar el cambio climático; cómo incrementar la actividad física en nuestros jóvenes; cómo mantener la producción de agua potable y reducir su contaminación; cómo sostener de manera eficiente la producción agrícola y reducir los gases de efecto invernadero. Se requiere, entre otras cosas, la definición de políticas nacionales y globales para reducir las emisiones de gases contaminantes, y que cada individuo y las comunidades aporten su granito de arena para reducir la contaminación del agua, preservar los bosques en busca de mantener las fuentes de agua y disminuir el consumo de energía.

La prensa escrita y las revistas científicas también enfrentan desafíos, entre ellos: cómo manejar el gran volumen de información que surge de la investigación que se realiza hoy en día; cómo dar al lector información actual y sencilla, con evidencia de buena calidad; cómo disminuir los costos de producción y cómo adaptarse a las nuevas tecnologías de la información y la comunicación (3). Las revistas de ciencias de la salud están evolucionando hacia la versión digital 
(12-14), la cual ofrece muchas más opciones para ser leídas por una mayor audiencia, por diferentes vías y dispositivos, que la versión en papel. formato no solo permite que se seleccione la información que interesa al lector, sino que además reduce los costos de producción y es amigable con el medio ambiente. La Revista Colombiana de Obstetricia y Ginecología ha migrado a la versión electrónica; mediante este cambio esperamos lograr una mayor audiencia y, al reducir los costos de publicación, utilizar esos recursos para poder presentar la información de acuerdo con los requerimientos actuales de reporte (15), de una manera sencilla para el lector y orientando al mismo sobre la validez de los resultados presentados. Esperamos poder seguir contando con los manuscritos que informan los estudios desarrollados por sus grupos.

\section{Hernando Gaitán-Duarte, MD, MSc}

Editor

\section{REFERENCIAS}

1. RCN radio. Secretario de Movilidad y gerentes de TM y UAESP, entre otros nuevos cargos, designa Enrique Peñalosa. [Visitado 2015 Dic 10]. Disponible en https://www.rcnradio.com/locales/bogota/asi-se-vaconformando-gabinete-enrique-penalosa/

2. Universia España. Tendencias tecnológicas de los estudiantes. 18 de marzo de 2014. [Visitado 2015 Dic 10]. Disponible en: http://noticias.universia.es/cienciann-tt/noticia/2014/03/18/1088517/tendenciastecnologicas-estudiantes.html

3. Nagendran M, Dimick JB. Disseminating research findings: preparing for Generation Y. JAMA Surg. 2014;149:629-30.

4. In US, 5\% Consider Themselves Vegetarians. [Visitado 2015 Dic 10]. Disponible en: http://www.gallup.com/ poll/156215/consider-themselves-vegetarians.aspx.

5. Spengler S, Mess F, Woll A. Do Media Use and Physical Activity Compete in Adolescents? Results of the MoMo Study. PLoS One. 2015;10:e0142544. doi: 10.1371/ journal.pone.0142544. eCollection 2015.
6. Wong BY, Cerin E, Ho SY, Mak KK, Lo WS, Lam TH. Adolescents' physical activity: competition between perceived neighborhood sport facilities and home media resources. Int J Pediatr Obes. 2010;5:169-76.

7. Spengler S, Mess F, Schmocker E, Woll A. Longitudinal associations of health-related behavior patterns in adolescence with change of weight status and self-rated health over a period of 6 years: results of the MoMo longitudinal study. BMC Pediatr. 2014;14:242. doi: 10.1186/1471-2431-14-242.

8. IPCC. Climate Change 2014: Synthesis Report, Contribution of Working Groups I, II and III to the Fifth Assessment Report of the Intergovernmental Panel on Climate Change. En: Pachauri RK, Meyer LA, editors. Core Writing Team. Geneva: IPCC; 2014. p. 151.

9. Robinet C, Laparie M, Rousselet J. Looking Beyond the Large Scale Effects of Global Change: Local Phenologies Can Result in Critical Heterogeneity in the Pine Processionary Moth. Front Physiol. 2015;6:334. eCollection 2015

10. Caroselli E, Falini G, Goffredo S, Dubinsky Z, Levy O. Negative response of photosynthesis to natural and projected high seawater temperatures estimated by pulse amplitude modulation fluorometry in a temperate coral. Front Physiol. 2015;6:317.

11. Averting climate change's health effects in Fiji. Bull World Health Organ. 2015;93:746-7.

12. JAMA. Multimedia. [Visitado 2015 Dic 10]. Disponible en: http://jama.jamanetwork.com/multimedia.aspx.

13. American Journal of Obstetrics and Gynecology [on line]. [Visitado 2015 Dic 10]. Disponible en: https://secure.jbs.elsevierhealth.com/action/ activateClaim ? code $=$ ymob-site

14. British Journal Obstetrics \& Gynecol [on line]. [Visitado 2015 Dic 10]. Disponible en: http://onlinelibrary.wiley. com/journal/10.1111/\%28ISSN\%291471-0528/ issues .

15. Rubio-Romero JA. La Revista Colombiana de Obstetricia y Ginecología y los estándares internacionales de publicación en revistas científicas. Rev Colomb Obstet Ginecol. 2014;65:8-10. 